\title{
Chromatin interaction maps identify Wnt responsive cis-regulatory elements coordinating Paupar-Pax6 expression in neuronal cells
}

loanna Pavlaki ${ }^{1 *}$, Michael Shapiro ${ }^{1,2}$, Giuseppina Pisignano ${ }^{1}$, Jelena Telenius ${ }^{3}$, Silvia Muñoz Descalzo ${ }^{1,4}$, Robert J. Williams ${ }^{1}$, Jim R. Hughes ${ }^{3}$ and Keith W. Vance ${ }^{1 \#}$

${ }^{1}$ Department of Biology and Biochemistry, University of Bath, Bath, BA2 7AY, UK

${ }^{2}$ The Francis Crick Institute, London, NW1 1AT, UK

${ }^{3}$ MRC Molecular Haematology Unit, Weatherall Institute of Molecular Medicine, Radcliffe Department of Medicine, University of Oxford, Oxford, UK

${ }^{4}$ Instituto Universitario de Investigaciones Biomédicas y Sanitarias, Universidad Las Palmas de Gran Canaria, Las Palmas de Gran Canaria, Spain

\#To whom correspondence should be addressed. Tel: +44 (0)1225 385106; Email:

k.w.vance@bath.ac.uk 


\begin{abstract}
Central nervous system-expressed long non-coding RNAs (IncRNAs) are often located in the genome close to protein coding genes involved in transcriptional control. Such IncRNA-protein coding gene pairs are frequently temporally and spatially co-expressed in the nervous system and are predicted to act together to regulate neuronal development and function. Although some of these IncRNAs also bind and modulate the activity of the encoded transcription factors, the regulatory mechanisms controlling co-expression of neighbouring IncRNA-protein coding genes remain unclear. Here, we used high resolution NG Capture-C to map the cis-regulatory interaction landscape of the key neurodevelopmental Paupar-Pax 6 IncRNA-mRNA locus. The results defined chromatin architecture changes associated with high Paupar-Pax 6 expression in neurons and identified both promoter selective as well as shared cis-regulatory interactions with the Paupar and Pax6 promoters involved in regulating Paupar-Pax6 co-expression in neuronal cells. The TCF7L2 transcription factor, a major regulator of chromatin architecture and effector of the Wnt signalling pathway, binds to a subset of these candidate cis-regulatory elements to coordinate Paupar and Pax6 co-expression. We identify a functional TCF7L2 bound cis-regulatory element within the Paupar gene, suggesting that the Paupar DNA locus itself regulates Pax6 expression in addition to its previously described transcriptdependent modes of action. Our work provides important insights into the chromatin interactions, signalling pathways and transcription factors controlling co-expression of adjacent IncRNAs and protein coding genes in the brain.
\end{abstract}




\section{Introduction}

A typical gene promoter is regulated by multiple different types of cis-regulatory elements (CREs) such as transcriptional enhancers and silencers. These are DNA sequences containing clusters of transcription factor binding sites that act together to generate the correct temporal and spatial expression of their target genes (Jeziorska, Jordan et al. 2009). Chromatin conformation capture (3C) based technologies have shown that short- and long-range dynamic chromatin looping interactions bring CREs and their target promoters into close physical proximity in the nucleus to facilitate gene regulation. More recently, high throughput $3 \mathrm{C}$ variants such as NG Capture-C have been used to map large numbers of CREs to their cognate genes at high resolution and investigate the complexity of CRE-promoter communication at unprecedented detail (Davies, Telenius et al. 2016, Davies, Oudelaar et al. 2017).

Precise temporal and spatial control of expression of the Pax 6 transcription factor gene is required for the normal development and function of the nervous system. Pax6 haploinsufficiency in mice results in abnormal eye and nasal development and causes a range of brain defects; whilst mutations affecting PAX6 expression and function in humans cause anirida, an autosomal dominant inherited disorder characterized by a complete or partial absence of the iris (Jordan, Hanson et al. 1992, Manuel, Mi et al. 2015, Lima Cunha, Arno et al. 2019). Pax6 is transcribed from 2 major upstream promoters (PO, P1) and multiple CREs have been shown to control Pax6 expression in distinct domains in the central nervous system and eye (Williams, Altmann et al. 1998, Xu, Zhang et al. 1999, Morgan 2004). These include the neuroretina, ectodermal and retinal progenitor enhancers just upstream of the P0 promoter (Kammandel, Chowdhury et al. 1999, Plaza, Saule et al. 1999); a photoreceptor enhancer situated between the P0 and P1 promoters ( $\mathrm{Xu}$, Zhang et al. 1999); the retina regulatory region located between exons 4 and 5 (Kammandel, Chowdhury et al. 1999, Xu, Zhang et al. 1999); and three conserved sequence elements within intron 7 that activate Pax6 in the diencephalon, rhombencephalon and at late stages of eye development (Kleinjan, Seawright et al. 2004). These CREs are all located within a $30 \mathrm{~kb}$ window surrounding the Pax6 P0 and P1 promoters and act over short genomic distances. In addition, several candidate long-range enhancers have been identified approximately 150-200 kb downstream of the Pax6 gene and some of these have also been shown to drive Pax 6 expression in specific domains of the eye and brain (Kleinjan, Seawright et al. 2006, McBride, Buckle et al. 2011). However, these enhancers together are not sufficient to generate the full temporal and spatial pattern of Pax6 expression in the central nervous system suggesting the presence of additional uncharacterised Pax6 regulatory elements.

Thousands of IncRNAs are temporally and spatially expressed within the central nervous system and some of these are thought to be important in brain development and function (Derrien, Johnson et 
al. 2012, Liu, Nowakowski et al. 2016, Hezroni, Ben-Tov Perry et al. 2020). Brain-expressed IncRNAs are preferentially located in the genome close to protein coding genes involved in transcriptional control (Ponjavic, Oliver et al. 2009). This includes bidirectional IncRNAs that are transcribed in the opposite direction to a protein coding gene from a shared promoter as well as intergenic IncRNAs that are either expressed from their own promoter or from a transcriptional enhancer. Such IncRNAmRNA pairs are frequently co-expressed during neuronal development and in different brain regions and can function in the control of similar biological processes (Chalei, Sansom et al. 2014, Vance, Sansom et al. 2014). The IncRNA Paupar, transcribed from a promoter approximately $8.5 \mathrm{~kb}$ upstream of the Pax6 gene, is an important regulator of neurogenesis in vivo in mouse and human, and is co-ordinately expressed with Pax6 during neural differentiation in vitro and in the adult mouse brain (Vance, Sansom et al. 2014, Pavlaki, Alammari et al. 2018, Xu, Xi et al. 2021). Moreover, Paupar transcript directly binds PAX6 and acts as a transcriptional cofactor to promote the formation of a PAX6-Paupar-KAP1 chromatin regulatory complex at important neuronal genes (Vance, Sansom et al. 2014, Pavlaki, Alammari et al. 2018). Even though Paupar and Pax6 can act together to regulate shared biological processes important for neuronal development, the CRES controlling Paupar-Pax6 co-expression in the nervous system are not known.

Here we used NG Capture-C to generate high resolution chromatin interaction maps with the Paupar and Pax6 promoters in Paupar-Pax6 high- and low-expressing cells. The results identified shared chromatin interactions with both the Paupar and Pax6 promoters involved in regulating PauparPax6 co-expression, as well as promoter specific cis-regulatory interactions. We show that the WntBmp4 signalling axis acts through the TCF7L2 transcription factor to co-ordinate Paupar and Pax6 coexpression in neuronal cells, including through a functional TCF7L2 bound cis-regulatory element within the Paupar gene. We report cell type specific differences in both local and distal chromatin interactions with the Paupar and Pax6 promoters that may be important for CRE-promoter communication and Paupar-Pax6 activation in neurons. Our work further refines the complex cisregulatory landscape surrounding the Paupar-Pax6 locus and provides critical insights into the regulatory mechanisms controlling the co-expression of adjacent IncRNAs and protein coding genes in the brain. 


\section{Results}

\section{Identification of cis-regulatory interactions with the Paupar and Pax6 promoters using high resolution NG Capture-C}

We previously showed that the IncRNA Paupar and its adjacent Pax6 transcription factor gene are highly expressed in the adult mouse brain, and that Paupar and Pax6 expression are temporally coordinated during in vitro neural differentiation of mouse embryonic stem cells (ESCs) (Vance, Sansom et al. 2014). In this study, we used the following neuronal cell types to investigate PauparPax6 expression control: primary neural stem cells (NSCs) isolated from E14.5 mice, differentiated mouse cortical neurons, N2A mouse neuroblastoma cells, as well as mouse ESCs as a non-neuronal reference. RT-qPCR analysis demonstrated that Paupar and Pax6 P0 and P1 expression is significantly higher in neuronal cell types compared to ESCs, with highest expression in NSCs and differentiated neurons (Fig. 1A, B). Consistent with an earlier report (Kleinjan, Seawright et al. 2001), our results also suggest that the Pax6 $\mathrm{P} 1$ promoter is the major Pax6 promoter in the neuronal lineage. We found that Pax6 P1 promoter transcription was 40- and 105-fold more active than P0 in NSCs and differentiated neurons respectively, whilst in N2A cells Pax6 P0 expression was undetectable (Fig. 1A, B).

High resolution NG Capture-C was performed to map chromatin interactions with the Paupar and Pax6 P0 and P1 promoters, as well as the Sox 2 promoter as a positive control, to identify the cisregulatory DNA sequences important for Paupar-Pax6 expression control and the chromatin changes associated with activation of the locus in neuronal cells. Multiplexed NG Capture-C libraries were generated and sequenced to an average depth of 61 million paired end reads per library.

Benchmarking NG Capture-C data quality using CCanalyser (Davies, Telenius et al. 2016) showed that an average of $37.6 \%$ mapped reads contained capture bait sequence across all NG Capture-C libraries, demonstrating good capture enrichment, and confirmed good ligation efficiency as $29.3 \%$ of captured fragments were ligated to a reporter (S1 Table). This enabled us to generate high resolution NG Capture-C interaction profiles (Fig. 2A, B, C, S1 Fig) using an average of 33,719 (S2 Table) unique interactions between Dpnll restriction fragments and each promoter bait fragment per cell type.

NG Capture-C interaction profiles were then analysed using r3C-seq tools (Thongjuea, Stadhouders et al. 2013) to normalise for distance from the capture point and model statistically significant ( $q<$ 0.1) CRE-promoter interactions. As expected, we identified significant looping interactions between the Sox2 super-enhancer overlapping the Peril locus (Li, Rivera et al. 2014) and the Sox2 promoter in ESCs which were not present in neuronal cell types (S1 Fig). This is consistent with previous $3 \mathrm{C}$ based 
maps defining Sox2 enhancer-promoter communication (Li, Rivera et al. 2014) and confirms the ability of our approach to identify functional CREs. To define the set of regulatory interactions mediating Paupar-Pax6 expression we then determined the number of statistically significant chromatin interactions with the Paupar and Pax6 P0 and P1 promoters present in both biological replicates for each cell type. We discovered that $96 \%$ high resolution chromatin interactions are located within a $50 \mathrm{~kb}$ window centred around each promoter (Fig $3 \mathrm{~A}$ ), including both upstream and downstream cis-acting DNA sequences. The Paupar-Pax6 cis-regulatory interaction map showed significant interactions with known Pax6 regulatory elements as well as many additional short-range regulatory interactions with candidate new CREs involved in Paupar-Pax6 expression control (Fig 3B and S3 Table for fragment coordinates). Consistent with a role in neuronal gene expression, chromatin interactions with the Paupar and Pax6 promoters show an increased overlap with H3K4me1 ChIP-seq peaks in E12.5 mouse forebrain tissue compared to ESCs using publicly available ENCODE data (He, Hariharan et al. 2020) (Fig 3B). The interaction map also encompasses associations between DNA sequence elements within the Paupar genomic locus and the Pax6 promoters (Fig 3B and S4 Table). Most chromatin interactions (119 out of 168) were found in all cell types tested but we also discovered a subset of neuronal (30/168) and ESC (19/168) specific interactions that may be important for tissue specific regulation of the locus ( $\mathrm{S} 5 \mathrm{Table}$ ). Our results revealed shared interactions with more than one promoter region that may be important for Paupar-Pax6 co-expression in the brain (Fig 3C). In addition, we found a subset of specific cisregulatory interactions with individual Paupar, $P a \times 6 P 0$ and $P 1$ promoter viewpoints, suggesting that Paupar and Pax6 expression control may be decoupled (Fig 3C), as well as a small number of transinteractions with DNA sequences on different chromosomes (Fig 3B and S3 Table). Altogether, these results identify the chromatin interactions and CREs that are likely to be important for precise Paupar-Pax6 expression control.

\section{Wnt-Bmp4 signalling acts through TCF7L2 to regulate co-expression of the Paupar-Pax6 locus}

We hypothesized that a subset of discovered genomic fragments would play a causal role in the formation of chromatin interactions needed for expression of the the Paupar-Pax6 locus and that these would have an elevated discovery rate in our analysis. N2A cells were used to identify and define the function of such sequences as these cells represent a well characterised tractable in vitro model of neuronal differentiation and were previously used to determine Paupar and Pax6 gene regulatory functions (Vance, Sansom et al. 2014, Pavlaki, Alammari et al. 2018). To identify sequences with increased proximity to the promoter viewpoints compared to surrounding sequence, we plotted the mean - $\log 10$ q-value for each reproducible cis-regulatory interaction against chromosome position using N2A cell NG Capture-C data. We next defined the Dpnll fragments with 
the highest local statistical significance and curated a subset of 42 unique fragments ( 24 for Paupar, 10 for Pax6 P0 and 22 for the Pax6 P1 viewpoint), visualised as peaks of increased statistical prevalence (Fig 3D). We then applied 4Cin (Irastorza-Azcarate, Acemel et al. 2018) to generate a 3D model of Paupar-Pax6 local chromatin architecture from N2A cell NG Capture-C data and visualise the relative proximity of the curated fragments to the Paupar and Pax6 promoters. The results showed that most curated fragments are located at curvature points on the chromatin fibre and appear to be orientated towards the Paupar-Pax6 promoters (Fig 3E). This subset of curated NG Capture-C fragments may represent candidate Paupar-Pax6 CREs with roles in the regulation of short-range chromatin interactions.

CRE-promoter communication is mediated by protein-protein interactions between transcription factors bound to specific motifs within CREs and proteins assembled at the target promoters. To investigate the transcription factors controlling Paupar-Pax6 co-expression in the brain we used the BiFa motif discovery tool (Jeziorska, Koentges et al. 2012) to search for transcription factor position frequency matrices (PFMs) within the N2A cell curated fragment dataset and identify putative binding sites for factors with known functions in neuronal development. This discovered 13 high scoring PFMs for the TCF7L2/TCF4 transcription factor (Table S6), an important regulator of chromatin structure and major effector of the $\mathrm{Wnt} / \beta$-catenin signalling pathway. To explore TCF7L2 regulation of Paupar-Pax6 we first determined whether Wnt signalling regulates expression of the Paupar-Pax6 locus. RT-qPCR analysis showed that ectopic expression of a constitutively active $\beta$ catenin S33Y protein (Morin, Sparks et al. 1997) significantly reduced both Paupar and Pax6 expression by $40 \%$ and $33 \%$ respectively in N2A cells (Fig $4 \mathrm{~A}$ ). In addition, activation of canonical Wnt signalling using recombinant WNT3a ligand led to a significant $39 \%$ reduction in $T c f 7 / 2$ expression and a concomitant 56\% reduction in Paupar and 47\% reduction in Pax6 levels in N2A cells after 72 hours (Fig 4B). As crosstalk between the Wnt and Bmp signalling pathways is important during neuronal development we then treated N2A cells with BMP4, a Wnt3a target gene in neuroblastoma (Szemes, Melegh et al. 2020). This led to a 2.34-fold increase in $T c f 7 / 2$ and a subsequent 2.13- and 1.89-fold up-regulation in Paupar and Pax6 expression after 72 hours (Fig 4C). Altogether, these results suggest that the Wnt-Bmp signalling axis acts through $T c f 712$ to coordinately regulate both Paupar and Pax6 expression in neural cells.

\section{Novel TCF7L2 bound CREs control Paupar-Pax6 co-expression}

We next investigated whether Tcf7/2 directly regulates Paupar-Pax6. To do this, ChIP-qPCR was performed to test whether TCF7L2 binds to any of its predicted motifs within the curated fragment dataset. We amplified 7 candidate CREs (CRE1-7) close to TCF7L2 motifs (Fig 4D) and identified 
strong (> 3-fold enrichment) TCF7L2 chromatin binding at three of them in N2A cells. TCF7L2 binding was 4-fold enriched at CRE2; 20-fold enriched at CRE3; and 11-fold enriched at CRE5, compared to an IgG isotype control (Fig 4E). We next tested whether TCF7L2 regulates Paupar and/or Pax6 expression. TC7FL2 levels were depleted in N2A cells using an endoribonuclease-prepared pool of TCF7L2 siRNAs (esiRNAs) and changes in Paupar and Pax6 expression measured using RT-qPCR (Fig $4 F)$. The results showed that silencing $T c f 7 / 2$ by approximately $76 \%$ resulted in a significant $50 \%$ reduction in Paupar and 18\% reduction in Pax6 expression. Taken together, these results suggest that TCF7L2 binds a subset of DNA sequence elements within NG Capture-C identified candidate CREs to directly activate both Paupar and Pax6 expression.

We then performed CRISPR interference (CRISPRi) to study the function of the TCF7L2 binding sites within their endogenous chromatin context in Paupar-Pax6 expression control. To do this, a single guide RNA (sgRNA) targeting a specific TCF7L2 motif was used to recruit a catalytically inactive dCas9-KRAB fusion protein to induce local chromatin closing and block regulatory element activity in N2A cells (Fulco, Munschauer et al. 2016). RT-qPCR analysis of Paupar and Pax6 expression showed that targeting dCas9-KRAB to CRE3 induced a significant 22\% reduction in Paupar and a 31\% reduction in Pax6 expression (Fig 4G, middle panel). CRISPRi against the TCF7L2 motif in CRE2 led to a 2.1-fold up-regulation of Paupar and a 1.4-fold increase in Pax6 expression (Fig 4G, left panel); whilst inhibition of CRE5, located within the Paupar DNA locus, resulted in a 2.0-fold increase in Paupar and a 1.5-fold increase in Pax6 expression (Fig 4G, right panel). These results suggest that the CRE3 TCF7L2 motif functions as part of a shared transcriptional enhancer of both Paupar and Pax6, and that the CRE2 and CRE5 TCF7L2 motif containing sequences co-ordinately repress both Paupar and Pax6. The Paupar DNA locus therefore appears to play a regulatory role in Pax6 expression control.

\section{Identification of cell type specific chromatin architecture changes associated with high Paupar and Pax6 expression in neurons}

Comparative analyses of NG Capture-C profiles have previously been used to investigate cisregulatory mechanisms controlling cell type specific gene expression (Davies, Telenius et al. 2016). We thus developed a new statistical method to compare the NG Capture-C profiles from PauparPax6 high-expressing differentiated neurons and low-expressing ESCs and detect changes in chromatin architecture associated with increased Paupar-Pax6 expression in the brain. In this, normalised NG Capture-C data were first grouped into bins of discrete sizes to increase signal over noise. The results revealed that a $10 \mathrm{~kb}$ bin size facilitated the identification of changes in chromatin conformation due to clustering of neighbouring fragments that were not significant at the individual 
fragment level (S2 Fig). Furthermore, these changes were not discovered using permuted data validating specificity.

Examination of changes in local chromatin architecture surrounding the Paupar-Pax6 locus $(+/-50 \mathrm{~kb}$ from each viewpoint) identified an increase in chromatin interactions upstream of the Pax6 gene with the Paupar promoter and an increase in downstream interactions with the Pax6 P1 promoter in neurons compared to ESCS, as well as a set of reciprocal interactions that increase in ESCs compared to neurons (Fig 5A). Consistently, permutation testing to compare the frequency of upstream versus downstream chromatin interactions from each viewpoint revealed significant asymmetry in chromatin architecture (Fig 5A). These changes in local chromatin organisation may be important for the rewiring of short-range CRE-promoter interactions controlling Paupar-Pax6 co-expression in neurons, and are consistent with ENCODE ChIP-seq data (He, Hariharan et al. 2020) showing that the chromatin surrounding the Paupar and Pax6 promoters is marked by an increase in open (H3K4me1, H3K4me3, H3K27ac) chromatin marks in E12.5 mouse forebrain tissue compared to ESCs (Fig 5B and S3 Fig).

As transcriptional regulatory elements can function over large genomic distances, we next analysed $1 \mathrm{MB}$ genomic sequence surrounding the Paupar-Pax6 locus using binned NG Capture-C data to map meso-scale changes in chromatin architecture between cell types. This detected a large up to $250 \mathrm{~kb}$ chromosomal region located approximately $350 \mathrm{~kb}$ downstream of the Pax6 gene that contains an increased frequency of statistically significant interactions with the Paupar and Pax6 promoters in differentiated neurons compared to ESCS (Fig 5A). This region corresponds to an equivalent region in the human genome containing multiple predicted long-range CREs that loop onto the human Pax6 promoter in Promoter Capture Hi-C experiments (Freire-Pritchett, Schoenfelder et al. 2017).

Furthermore, this region contains an increased number of H3K4me1, H3K4me3 and H3K27ac ChIPseq peaks (He, Hariharan et al. 2020) in mouse forebrain tissue compared to ESCs (Fig 5B and S3 Fig). As these histone modifications are known to mark active regulatory regions, we predict that this distal domain may contain additional clusters of uncharacterised long-range CREs involved in Paupar-Pax6 expression control in neuronal cells. Taken together, these data define the local and distal chromatin changes associated with Paupar-Pax6 expression in neurons. 


\section{Discussion}

LncRNAs involved in brain development are frequently co-expressed with their adjacent protein coding genes. These IncRNA-mRNA pairs often function in the same biological processes and some CNS expressed IncRNAs modulate both the expression and transcriptional activity of their neighbouring protein coding genes. A greater understanding of the complex regulatory relationship controlling the expression and function of InCRNA-mRNA pairs in the brain is needed to further define their role in neuronal development and function.

In this study we used high resolution NG Capture-C to comprehensively define chromatin interactions important for Paupar-Pax6 expression control. Our work revealed an intricate network of short-range cis-regulatory interactions with the Paupar and Pax6 P0 and P1 promoters, including interactions with the previously characterised Pax6 ectodermal, neuroretina, retinal progenitor and photoreceptor enhancers (Kammandel, Chowdhury et al. 1999, Plaza, Saule et al. 1999, Xu, Zhang et al. 1999), as well as many candidate new CREs. The results classified a subset of shared short-range chromatin interactions with both the Paupar and Pax6 promoters that are likely to be involved in regulating Paupar-Pax6 co-expression in the brain. We detected significant asymmetry in local chromatin architecture surrounding the Paupar and Pax6 $\mathrm{P} 1$ promoters and discovered a subset of short-range chromatin interactions with these promoters that increase in differentiated neurons compared to ESCs. We hypothesize that these chromatin changes are important for CRE-promoter communication and cell type specific expression of the locus.

Our results curated a subset of NG Capture-C fragments based on increased local statistical significance that may be central mediators of short-range CRE-promoter interactions. We discovered that TCF7L2 binds a subgroup of these fragments and acts to integrate signals from the Wnt and Bmp signalling pathways to control Paupar and Pax6 co-expression. Interplay between the Wnt and Bmp pathways is critical for proper development of the nervous system and in the Paupar-Pax 6 expressing subventricular zone (SVZ) NSC niche has been shown to regulate postnatal NSC selfrenewal and SVZ neurogenesis (Pavlaki, Alammari et al. 2018, Al-Dalahmah, Campos Soares et al. 2020, Al-Dalahmah, Nicholson et al. 2020). Furthermore, the Wnt-Bmp signalling axis also promotes growth suppression and differentiation in neuroblastoma (Szemes, Melegh et al. 2020). TCF7L2 is a key Wnt effector in the brain and is required for the production of Pax6 expressing neural progenitor cells in the neocortex (Chodelkova, Masek et al. 2018). We expect that TCF7L2 acts as an important regulator of Paupar-Pax6 chromatin organisation and CRE-promoter communication. Accordingly, TCF7L2 silencing leads to genome-wide changes in chromatin architecture and enhancer-promoter interactions in pancreatic and colon cancer cells, whilst TCF-bound Wnt responsive enhancers regulate chromatin looping and activation of the Myc gene in colorectal cancer (Yochum, Sherrick et 
al. 2010, Gerrard, Wang et al. 2019, Brown, Dotson et al. 2021). Furthermore, ENCODE data shows that TCF7L2 associates with more than $40 \%$ of active enhancers in the genome in different cell lines suggesting that TCF7L2 is a critical regulator of cell-type specific CRE function (ENCODE 2019). Our finding that Wnt3a-Bmp4 acts through TCF7L2 to co-ordinate Paupar-Pax6 co-expression in neuronal cells thus links Paupar-Pax6 co-expression control and chromatin regulation to important neurodevelopmental signalling pathways.

Our work also discovered a large chromatin domain downstream of the Pax6 gene that displays clusters of increased long-range chromatin interactions with the Paupar-Pax6 locus in E12.5 mouse neurons compared to ESCs. This region is further downstream from the previously defined Pax6 distal DRR and the aniridia-associated breakpoints within the last intron of the downstream ELP4 gene that are predicted to influence Pax6 expression in affected individuals (Kleinjan, Seawright et al. 2001, Kleinjan, Seawright et al. 2006). However, it maps to an equivalent region in the human genome that contains multiple long-range enhancer-promoter looping interactions with the Pax6 promoter, is characterised by an increase in enhancer-like chromatin modifications and is located with within the same self-interacting TAD as the Paupar and Pax6 promoters in neurons (Bonev, Mendelson Cohen et al. 2017, Freire-Pritchett, Schoenfelder et al. 2017, Lu, Liu et al. 2020). Our results are thus consistent with a model in which developmentally regulated changes in distal chromatin architecture also play a role in CRE-promoter rewiring and the activation of Paupar and Pax6 expression in the neuronal lineage.

Paupar IncRNA represses Pax6 expression and can modulate Pax6 splicing in a transcript- and cell type-dependent manner (Vance, Sansom et al. 2014, Singer, Arnes et al. 2019). Our results here report additional functions for the Paupar DNA locus in Paupar-Pax6 expression control. NG Capture-C revealed reproducible cis-regulatory interactions between DNA sequences within the Paupar locus and the Pax6 P0 promoter in neuronal cell types, consistent with phase III ENCODE data showing that Paupar overlaps five candidate CREs with an enhancer-like chromatin signature (Consortium, Moore et al. 2020). We found that TCF7L2 binds to a functional silencer element, termed CRE5, within the Paupar locus and that recruitment of the dCas9-KRAB chromatin repressor to the TCF7L2 motif led to an increase in both Paupar and Pax6 expression. dCas9-KRAB recruitment is not predicted to directly block Paupar transcription (Gilbert, Horlbeck et al. 2014), as the targeted TCF7L2 motif lies approximately $2 \mathrm{~kb}$ downstream of the Paupar TSS, suggesting that the capacity of CRE5 to repress both Paupar and Pax6 expression is independent of the Paupar transcript produced. Similarly, several studies have reported distinct roles for IncRNA transcripts and transcriptional regulatory elements within their DNA loci in gene expression control. The Haunt DNA locus contains several transcriptional enhancer elements that loop onto the HoxA gene to increase its expression 
whereas the Haunt transcript binds upstream of HoxA to induce a repressive chromatin state and block HoxA expression (Yin, Yan et al. 2015). Tug1 DNA contains a CRE that represses multiple neighbouring downstream genes whilst the Tug1 IncRNA acts in trans to regulate different genes (Lewandowski, Dumbovic et al. 2020).

The identification of shared Paupar and Pax6 CREs also raises the possibility that the Paupar promoter may be able to control Pax6 expression through CRE competition as described for several other IncRNAs. The promoters of the Pvt1 IncRNA and neighbouring Myc oncogene compete for interactions with four shared enhancers. Silencing the Pvt1 promoter using CRISPRi increased enhancer contacts with the Myc promoter and up-regulated Myc expression independent of the Pvt1 transcript (Cho, Xu et al. 2018). Similarly, the Handsdown locus interacts with several enhancers for the adjacent Hand 2 gene and regulates their usage during cardiac differentiation (Ritter, Ali et al. 2019). Our study provides significant new insights into the chromatin interactions, transcription factors and signalling pathways controlling Paupar-Pax6 co-expression in the neuronal lineage and has general importance for understanding the wider role of IncRNA-mRNA transcription units in neuronal commitment, differentiation and function. 


\section{Materials and Methods}

\section{Plasmids}

Individual sgRNAs were cloned into $\mathrm{pX}$-dCas9-mod-KRAB to generate plasmids for CRISPRi as described in (Coe, Tan et al. 2019). Oligonucleotides used to clone sgRNAs targeting the TCF7L2 motif in CRE2, CRE3 and CRE5 are shown in S7 Table. The plasmid pCl-neo beta catenin S33Y was a gift from Bert Vogelstein (Addgene plasmid \# 16519; http://n2t.net/addgene:16519; RRID: Addgene_16519).

\section{Cell culture}

Primary cortical neurons and cortical neural stem cells were prepared from CD1 mouse embryos (E14.5) in accordance with UK Home Office Guidelines as stated in the Animals (Scientific Procedures) Act 1986 using Schedule 1 procedures approved by the University of Bath Animal Welfare and Ethical Review Body. Cortices were dissected from embryonic brain and mechanically dissociated in PBS supplemented with 33 mM glucose, using a fire-polished glass Pasteur pipette.

Primary neurons: For preparation of differentiated cortical neurons (Cox, Choudhry et al. 2015), cells were plated into Nunc $90 \mathrm{~mm}$ petri dishes, previously coated with $20 \mu \mathrm{g} / \mathrm{ml}$ poly-D-lysine (Sigma), at a seeding density of $500 \times 10^{5}$ cells/ml. Neurons were cultured in Neurobasal medium (phenol red free) supplemented with $2 \mathrm{mM}$ glutamine, $100 \mu \mathrm{g} / \mathrm{ml}$ penicillin, $60 \mu \mathrm{g} / \mathrm{ml}$ streptomycin and B27 (all from Gibco), and incubated at $37^{\circ} \mathrm{C}$, in high humidity with $5 \% \mathrm{CO}_{2}$. Under these growth conditions at 7 days in vitro (DIV) cells were non-dividing, had a well-developed neuritic network and were $99 \% \beta$ tubulin III positive and $<1 \%$ GFAP positive.

Primary neural stem cells: For preparation of cortical neural stem cells (Molina-Holgado, Rubio-Araiz et al. 2007), cells were plated into Nunc $90 \mathrm{~mm}$ petri dishes, previously coated with Cell Start (Gibco), at a seeding density of $500 \times 10^{5}$ cells $/ \mathrm{ml}$. Neural stem cells were cultured in StemPro NSC SFM composed of: Knockout D-MEM / F12; Glutamax (2mM); bFGF (20ng/ml); EGF (20ng/ml); StemPro Neural Supplement (2\%); all from (Gibco). Under these growth conditions at 7 DIV cells were proliferative and were Nestin and Ki67 positive.

Cell Lines: N2A cells were grown in DMEM supplemented with $10 \%$ fetal bovine serum (FBS). For the mouse ESCs experiments E14Tg2A cells were maintained in GMEM supplemented with $10 \%$ FBS, 1xMEM nonessential amino acids, $2 \mathrm{mM}$ glutamax, $1 \mathrm{mM}$ sodium pyruvate, $100 \mathrm{mM} 2-$ mercaptoethanol, and 100 units/ml LIF on gelatinised tissue culture flasks.

\section{Transfections and treatments}


Approximately $3 \times 10^{5} \mathrm{~N} 2 \mathrm{~A}$ cells were seeded per well in a 6-well plate for both plasmid DNA and esiRNA transfections. The following day, cells were transiently transfected using Lipofectamine 2000 (Invitrogen) following the manufacturer's instructions. For knockdown experiments, cells were transfected with $1.5 \mu \mathrm{g}$ MISSION ${ }^{\circledR}$ esiRNA (SIGMA-ALDRICH) targeting either TCF7/2 (EMU010891) or Renilla Luciferase (EHURLUC) control and harvested 3 days later. $2 \mu \mathrm{g} \mathrm{pCl-neo} \mathrm{beta} \mathrm{catenin} \mathrm{S33Y} \mathrm{or}$ empty vector were used in B-catenin S33Y overexpression experiments and cells were harvested 48 hrs post transfection. CRISPRi experiments were carried out as described in (Coe, Tan et al. 2019).

For Wnt and Bmp treatment, approximately $3 \times 10^{5} \mathrm{~N} 2 \mathrm{~A}$ cells were seeded per well in a 6-well plate in either growth medium containing $50 \mathrm{ng} / \mu \mathrm{lWnt3a}$ (R\&D Biosystems, 5036-wn) or in low serum medium (DMEM supplemented with 5\% FBS) containing $0.1 \mathrm{ng} / \mu \mathrm{l} \mathrm{BMP4}$ (Thermo Fisher, PHC9534) as described in (Szemes, Melegh et al. 2020). 0.02\% BSA in PBS was used as a vehicle control. Cells were harvested for RNA extraction 72 hours later. Sequences of primers used for expression analysis are shown in Table S7.

\section{NG Capture-C}

NG Capture-C libraries were prepared as described previously (Davies, Telenius et al. 2016). Briefly, approximately $2 \times 10^{7}$ cells per sample were fixed with $2 \%$ formaldehyde for $10 \mathrm{~min}$ at RT, quenched by the addition of glycine and washed with PBS. Cell lysis was performed for $20 \mathrm{~min}$ on ice $10 \mathrm{mM}$ Tris- $\mathrm{HCl}, \mathrm{pH} 8,10 \mathrm{mM} \mathrm{NaCl}, 0.2 \%$ IGEPAL), lysed cells were then homogenized on ice and enzymatically digested overnight at $37^{\circ} \mathrm{C}$ with Dpnll (New England Biolabs). The digested DNA was diluted and ligated with T4 DNA ligase overnight at $16^{\circ} \mathrm{C}$. The following day, ligation reactions were de-crosslinked by Proteinase $\mathrm{K}$ (Thermo Scientific) addition and overnight incubation at $65^{\circ} \mathrm{C}$. DNA extraction was performed by $\mathrm{PCl}$ and chloroform extraction followed by ethanol precipitation. Dpnll digestion efficiency was confirmed by gel electrophoresis and quantified by real-time PCR - only $3 C$ libraries with over $70 \%$ efficiency were used for the subsequent steps. Samples were sonicated to an average size of 200 bp using a Bioruptor Pico (Diagenode) and NEBNext Multiplex reagents and sequencing adapters were used to prepare sequencing libraries following the Illumina NEBNext DNA library prep kit instructions (New England Biolabs). Two rounds of capture using a pool of biotinylated oligos (IDT, see Table S7 for sequences) were performed on 1ug of each of the indexed libraries using the Nimblegen SeqCap EZ hybridization system. Library size was determined using the Tapestation D1000 kit and the DNA concentrations were measured on a Qubit 2.0 Fluorometer.

\section{Computation analysis of NG Capture-C data}

Multiplexed NG Capture-C libraries were prepared from ESCs, NSCS, neurons and N2A cells (two biological replicates each) and 150 bp paired end sequencing was performed on the lllumina HiSeq 
4000 (Novogene) to a total depth of approximately 500 million reads. The resulting fastq files from each of the eight replicates were combined using a Perl script. Raw reads were trimmed using trim_galore version 0.4.4 with parameter -paired. The trimmed paired-end reads were then combined using flash version 1.2.11 with the parameters --interleaved-output -max-overlap $=200$.

The resulting fastq files of combined and uncombined reads were next merged into a single fastq file using the command cat. The fragments in the resulting fastq file were in silico digested into Dpnll restriction enzyme digestion fragments using dpnII2E.pl. The resulting dpnIIE fragments were aligned to the $\mathrm{mm} 10$ genome using bowtie version 1.1 .2 with parameters $-\mathrm{p} 1$-m 2 --best --strata --chunkmb 256 -sam. A set of Dpnll fragments for the full mouse genome was produced from mm10.fa using gpngenome.pl. CCAnalyser3.pl was then run to compare a text file of the Paupar, Pax6 P0, Pax6 P1 and Sox 2 viewpoint coordinates with the in silico digested reads and genome to produce counts of the observed interactions with each viewpoint in each replicate. The output from CCAnalyser3.pl was analysed using the BioConductor $r 3 C$ seq package to determine statistical significance ( $p$ - and qvalues) for the observed interactions between the viewpoints and each Dpnll digestion fragment in each replicate. For each viewpoint, the resulting tables were combined into a table showing replicated significant fragments (significant in both replicates of at least one cell type) and these tables were used as the basis for subsequent analyses. Motif discovery was performed using the BiFa web tool at the Warwick Systems Biology Centre website (http://wsbc.warwick.ac.uk/wsbcToolsWebpage). 4Cin was used to generate three-dimensional models of Paupar-Pax6 local chromatin architecture (Irastorza-Azcarate, Acemel et al. 2018).

\section{A statistical method for detecting meso-scale changes in chromatin conformation from NG Capture-C data (DeltaCaptureC)}

We developed a new statistical method to detect changes in chromatin conformation based on significant clustering of neighbouring fragments from 3C-based data. This is available as a Bioconductor Software Package (10.18129/B9.bioc. deltaCaptureC). By binning NG Capture-C data and using permutation testing, this package can test whether there are statistically significant changes in the interaction counts between the data from two cell types or two treatments. To do this, read counts from the two biological replicates for each cell type were first combined. The counts for the four samples were normalised using DESeq2 function estimateSizeFactorsForMatrix() (Love, Huber et al. 2014) and the mean normalised count for both replicates in each cell type was determined. The difference between the two mean normalized counts was then calculated. This data was trimmed to a region of interest, $500 \mathrm{~kb}$ up- and down-stream of the midpoint of viewpoint, binned to a fixed bin size of $1 \mathrm{~kb}$ and then re-binned to $10 \mathrm{~kb}$ (S2 Fig). This identified a large distal region of increased chromatin interactions in neurons (Fig 5 and S2 Fig). We observed that this a 
contiguous region of constant sign (negative) with a combined total absolute value of 308.8. The null hypothesis is that this sum arises by chance. We tested this hypothesis to detect statistical significance for continuous regions of constant sign in the following manner: we first excluded the region $50 \mathrm{~kb}$ up- and downstream of the viewpoint and performed random permutation of the (nonviewpoint) $1 \mathrm{~kb}$ bins. After each such permutation, data was re-binned to $10 \mathrm{~kb}$ and each region was examined for constant sign. To do this, we computed its total absolute value and recorded the largest of these totals. If, after performing 1000 such random permutations, we observe fewer than 50 cases where the largest sum is 308.8 or greater, we have discovered a p-value for this region of less than 0.05 . In this way, we can exploit co-localisation of differences with like sign to detect meso-scale chromatin remodelling from 3C-based data (Fig 5A and S2 Fig).

We then considered the region near the viewpoint. In this case it is important to note that raw NG Capture-C counts in this region strongly correlate with distance from the viewpoint and we are thus unable to perform arbitrary permutation to test for statistical significance. However, performing permutations which do not change this distance allowed us to test the null hypothesis that chromatin remodelling flanking the viewpoint was symmetric. To this end, we computed the difference between the sum in the region upstream of the viewpoint and downstream of it using the actual data. We then computed this difference after multiple symmetric permutations. Since there are $501 \mathrm{~kb}$ bins in this region upstream and downstream of the viewpoint, there are $2^{50}$ permutations of this form giving us a sufficient number for permutation testing. In this way, we detected asymmetry in chromatin architecture in the neighbourhood of the Paupar and Pax6 promoter viewpoints in each cell type (Fig 5A).

\section{RT-qPCR}

RNA extraction was carried out using the GeneJET RNA Purification Kit (ThermoFisher) according to the manufacturer's instructions with the addition of an on-column DNase digestion step using the RNase-free DNase Set (QIAGEN). Reverse transcription was performed using the QuantiTect Reverse Transcription Kit (Qiagen). 1 mg total RNA was used in each reaction. Quantitative PCR was carried out on a Step One Plus Real-Time PCR System using Fast SYBR Green Master Mix (Applied Biosystems).

\section{Chromatin Immunoprecipitation}

ChIP experiments were performed as previously described using approximately $1 \times 10^{7} \mathrm{~N} 2 \mathrm{~A}$ cells per assay (Vance, Sansom et al. 2014). Cross-linked chromatin was immunoprecipitated with either $5 \mu \mathrm{g}$ anti-TCF4/TCF7L2 (Clone 6H5-3, \#05-511, Millipore) or normal mouse control lgG (\#12-371, 
bioRxiv preprint doi: https://doi.org/10.1101/2021.05.18.442939; this version posted May 18, 2021. The copyright holder for this preprint (which

was not certified by peer review) is the author/funder, who has granted bioRxiv a license to display the preprint in perpetuity. It is made available under aCC-BY-NC-ND 4.0 International license.

Millipore) antibodies. qPCR primers used to amplify TCF7L2 motif containing sequences (CRE1-7) are shown in S7 Table.

Data Availability

All multiplexed NG Capture-C DNA sequencing data for this project have been deposited in the NCBI GEO database (https://www.ncbi.nlm.nih.gov/geo/) under accession number GSE129697. 


\section{Figure Legends}

Figure 1. Paupar and Pax6 are co-ordinately expressed to higher levels in neuronal cells compared to ESCs.

(A) Genome browser graphic (GRCm38/mm10) showing the location of Paupar and Pax6 genes, the two major Pax6 promoters (P0 and P1) and known Pax6 enhancers. NR (neuroretina), EE (ectodermal enhancer), RP (retinal progenitors), PR (photoreceptor), Re (retina), CE1-3 (conserved element 1-3), HS (hypersensitivity site).

(B) Transcripts generated from the Paupar and Pax6 P0 and P1 promoters were measured in ESCs, NSCs, differentiated neurons and N2A cells using RT-qPCR. Results are presented relative to the Tbp reference gene. Mean values $+/$ - sem shown, $n=6$, one-tailed $t$-test, unequal variance. ${ }^{*} p<0.05,{ }^{* *}$ $p<0.01, * * * p<0.001$.

Figure 2. High resolution cis-regulatory interactions with the Paupar and Pax6 promoters in Paupar-Pax6 high- and low-expressing cells.

NG Capture-C profiles displaying the interaction count per Dpnll restriction enzyme fragment for each NG Capture-C library. The red vertical lines indicate the locations of captured viewpoints. (A) Paupar promoter, (B) Pax6 P0 and (C) Pax6 P1 promoter viewpoints. Significant fragments determined using R3C-seq (Thongjuea, Stadhouders et al. 2013) are denoted by coloured circles.

Figure 3. Identification of short-range regulatory interactions with candidate new CREs involved in Paupar-Pax6 expression control.

(A) Histogram depicting distances of cis-interacting fragments from their viewpoints. Significant duplicated fragments from all cell types and viewpoints are shown.

(B) Tables showing the number of statistically significant reproducible interactions with the indicated promoter viewpoints as well as overlap with known Pax6 enhancers and enhancer-like chromatin marks using ENCODE data (He, Hariharan et al. 2020).

(C) Venn diagram displaying the number of shared and specific interactions with the Paupar, Pax6 P0 and Pax6 P1 promoters in NSCs (left), neurons (middle) and N2A cells (right).

(D) Curation of interacting fragments for the indicated viewpoints based on combined $-\log 10 q$ value from NG Capture-C N2A cell data. q values from replicates were merged by taking the square root of their product. Each bar represents a fragment with significant interaction $(q<0.05)$. 
(E) 3D model of Paupar-Pax6 local chromatin architecture generated from NG Capture-C N2A cell data using the 4Cin software package (Irastorza-Azcarate, Acemel et al. 2018). Viewpoints and curated fragments are marked using the designated colours. The model is shown from three angles.

Figure 4. Wnt-Bmp signalling acts through TCF7L2 to coordinate Paupar-Pax6 expression in neuronal cells.

(A) N2A cells were transfected with $\mathrm{pCl} \beta$-catenin $\mathrm{S} 33 \mathrm{Y}$ or $\mathrm{pCl}$ empty vector and harvested for expression analysis 2 days later. Results are presented relative to the $\mathrm{pCl}$ control.

(B, C) N2A cells were treated with either $50 \mathrm{ng} / \mu \mathrm{l} \mathrm{Wnt3a(B)}$ or $0.1 \mathrm{ng} / \mu \mathrm{l} \mathrm{BMP4}$ (C) and harvested for RT-qPCR analysis 3 days later. $0.02 \%$ BSA in PBS was used as a negative control.

(D) Genome browser graphic showing the ChIP amplified candidate CREs as well as the location of the TCF7L2 motifs and sgRNAs used in the CRISPRi experiment (GRCm38/mm10).

(E) ChIP assays were performed in N2A cells using either an antibody against TCF7L2 or an isotype specific control. TCF7L2 occupancy at the indicated CREs was analysed by qPCR. Fold enrichment was calculated as $2^{-\Delta \Delta C t}(I P / \operatorname{lgG})$ and is presented as mean value $+/-$ sem. $n \geq 3$. One-tailed student's t-test $* p<0.05$.

(F) TCF7L2 levels were knocked-down in N2A cells by transfection of an endoribonuclease-prepared pool of TCF7L2 esiRNAs. An esiRNA pool targeting the luciferase gene was used as a negative control. Cells were harvested for expression analysis 3 days after transfection.

(G) N2A cells were transfected with a plasmid co-expressing dCas9-KRAB and a sgRNA targeting the TCF7L2 motif within the indicated candidate CREs. A non-targeting sgRNA was used as a control.

For all RT-qPCR reactions: Tcf7/2, Paupar and Pax6 expression levels were measured using RT-qPCR and results were normalised to Tbp. Results are presented as mean values $+/-$ sem, $n \geq 3$. One-tailed student's t-test $* p<0.05,{ }^{* *} p<0.01,{ }^{* * *} p<0.001$.

Figure 5. Local and distal chromatin changes associated with increased Paupar-Pax6 expression in neuronal cells.

(A) A comparative analysis of NG Capture-C data to map changes in chromatin conformation between cell types. Differences in mean normalized capture counts for interactions with the Paupar (top), Pax6 P0 (middle) and Pax6 P1 (bottom) viewpoints between ESC and neurons are plotted on the $y$-axis. X-axis shows position on chromosome 2 (GRCm38/mm10). Data are binned to $10 \mathrm{~kb}$ 
across approximately $\pm 500 \mathrm{~kb}$ genomic sequence around each viewpoint. Permutation testing was performed to determine statistical significance as described in Materials and Methods.

(B) ENCODE Project ChIP-seq data mapping the location of H3K4me1, H3K4me3 and H3K27ac peaks in ESCs and mouse forebrain tissue across approximately $1 \mathrm{MB}$ genomic sequence surrounding the Paupar-Pax6 locus (He, Hariharan et al. 2020). Individual peaks of less than $1 \mathrm{~kb}$ are shown at $1 \mathrm{~kb}$ long for visibility reasons.

\section{Supporting Information Legends}

Figures

S1 Fig. NG Capture-C identified ESC-specific chromatin looping interactions between the Sox2 super-enhancer spanning the Peril locus and the Sox2 promoter.

NG Capture-C profiles displaying the Sox2 promoter interaction count per Dpnll restriction enzyme fragment in the indicated cell types. The red vertical line indicates the location of the Sox 2 promoter viewpoint. Significant interactions were determined using R3C-seq

S2 Fig. Increasing bin size facilitates the detection of chromatin changes between cell types.

Differences in mean normalized NG Capture-C counts between neurons and ESCs for interactions with the Paupar viewpoint are plotted on the $y$-axis. X-axis shows position on chromosome 2 (GRCm38/mm10). Sequence data was permuted to assess specificity and statistical significance was calculated as described in Materials and Methods.

(A) Difference in mean normalised interactions between ESCs and neurons binned to $1 \mathrm{~kb}$. Negative values shown in red indicate increased interactions in neurons.

(B) The same data binned to $10 \mathrm{~kb}$. Note the emergence of the large red region approximately 350 $\mathrm{kb}$ downstream of Pax6 and the asymmetric pattern near the viewpoint.

(C) The $1 \mathrm{~kb}$ bins from (A) permuted. Bins further than $50 \mathrm{~kb}$ from the viewpoint are permuted at random. Bins closer than $50 \mathrm{~kb}$ are only permuted keeping their distance from the viewpoint.

(D) The previous panel re-binned to $10 \mathrm{~kb}$. Notice that no large contiguous regions of constant sign appear, nor does the asymmetric pattern seen near the viewpoint in (B).

S3 Fig. An increased frequency of chromatin interactions with the Paupar and Pax6 promoters in neurons correlates with elevated levels of open compared to closed histone modifications. 
ENCODE Project ChIP-seq data mapping the location of open (H3K4me1, H3K4me3 and H3K27ac) and closed (H3K27me3 and H3K9me3) ChIP-seq peaks in ESCS (A) and E12.5 mouse forebrain tissue (B) across approximately $1 \mathrm{MB}$ genomic sequence surrounding the Paupar-Pax6 locus (He, Hariharan et al. 2020). Individual peaks of less than $1000 \mathrm{bp}$ are shown at $1000 \mathrm{bp}$ long for visibility reasons. Aggregated data represent summed and binned scores from the individual tracks.

Tables

S1 Table. Determination of ligation and capture frequencies for NG Capture-C experiment

S2 Table. Number of unique interactions with each promoter viewpoint

S3 Table. Genome coordinates of significant replicated fragments for each viewpoint (Mouse GRCm38/mm10)

S4 Table. Interactions between the indicated viewpoints and reporter fragments that overlap the Paupar genomic locus

S5 Table. Number of replicated interactions in different cell types

S6 Table. BiFa analysis identification of TCF7L2 motifs

S7 Table. Oligonucleotides 
bioRxiv preprint doi: https://doi.org/10.1101/2021.05.18.442939; this version posted May 18, 2021. The copyright holder for this preprint (which

was not certified by peer review) is the author/funder, who has granted bioRxiv a license to display the preprint in perpetuity. It is made available under aCC-BY-NC-ND 4.0 International license.

\section{Acknowledgements}

This project has been funded by a Biotechnology and Biological Sciences Research Council grant to KWV (BB/N005856/1; KWV, IP, MS). We thank Dr Karim Malik (University of Bristol) for providing recombinant Wnt3a and Bmp4. 


\section{References}

Al-Dalahmah, O., L. Campos Soares, J. Nicholson, S. Draijer, M. Mundim, V. M. Lu, B. Sun, T. Tyler, I. Adorjan, E. O'Neill and F. G. Szele (2020). "Galectin-3 modulates postnatal subventricular zone gliogenesis." Glia 68(2): 435-450.

Al-Dalahmah, O., J. Nicholson, S. Draijer, L. C. Soares and F. G. Szele (2020). "Galectin-3 diminishes Wnt signaling in the postnatal subventricular zone." Stem Cells 38(9): 1149-1158.

Bonev, B., N. Mendelson Cohen, Q. Szabo, L. Fritsch, G. L. Papadopoulos, Y. Lubling, X. Xu, X. Lv, J. P. Hugnot, A. Tanay and G. Cavalli (2017). "Multiscale 3D Genome Rewiring during Mouse Neural Development." Cell 171(3): 557-572 e524.

Brown, M. A., G. A. Dotson, S. Ronquist, G. Emons, I. Rajapakse and T. Ried (2021). "TCF7L2 silencing results in altered gene expression patterns accompanied by local genomic reorganization." Neoplasia 23(2): 257-269.

Chalei, V., S. N. Sansom, L. Kong, S. Lee, J. F. Montiel, K. W. Vance and C. P. Ponting (2014). "The long non-coding RNA Dali is an epigenetic regulator of neural differentiation." Elife 3: e04530.

Cho, S. W., J. Xu, R. Sun, M. R. Mumbach, A. C. Carter, Y. G. Chen, K. E. Yost, J. Kim, J. He, S. A. Nevins, S. F. Chin, C. Caldas, S. J. Liu, M. A. Horlbeck, D. A. Lim, J. S. Weissman, C. Curtis and H. Y. Chang (2018). "Promoter of IncRNA Gene PVT1 Is a Tumor-Suppressor DNA Boundary Element." Cell 173(6): 1398-1412 e1322.

Chodelkova, O., J. Masek, V. Korinek, Z. Kozmik and O. Machon (2018). "Tcf7L2 is essential for neurogenesis in the developing mouse neocortex." Neural Dev 13(1): 8.

Coe, E. A., J. Y. Tan, M. Shapiro, P. Louphrasitthiphol, A. R. Bassett, A. C. Marques, C. R. Goding and K. W. Vance (2019). "The MITF-SOX10 regulated long non-coding RNA DIRC3 is a melanoma tumour suppressor." PLOS Genet 15(12): e1008501.

Consortium, E. P., J. E. Moore, M. J. Purcaro, H. E. Pratt, C. B. Epstein, N. Shoresh, J. Adrian, T. Kawli, C. A. Davis, A. Dobin, R. Kaul, J. Halow, E. L. Van Nostrand, P. Freese, D. U. Gorkin, Y. Shen, Y. He, M. Mackiewicz, F. Pauli-Behn, B. A. Williams, A. Mortazavi, C. A. Keller, X. O. Zhang, S. I. Elhajjajy, J. Huey, D. E. Dickel, V. Snetkova, X. Wei, X. Wang, J. C. Rivera-Mulia, J. Rozowsky, J. Zhang, S. B. Chhetri, J. Zhang, A. Victorsen, K. P. White, A. Visel, G. W. Yeo, C. B. Burge, E. Lecuyer, D. M. Gilbert, J. Dekker, J. Rinn, E. M. Mendenhall, J. R. Ecker, M. Kellis, R. J. Klein, W. S. Noble, A. Kundaje, R. Guigo, P. J. Farnham, J. M. Cherry, R. M. Myers, B. Ren, B. R. Graveley, M. B. Gerstein, L. A. Pennacchio, M. P. Snyder, B. E. Bernstein, B. Wold, R. C. Hardison, T. R. Gingeras, J. A.

Stamatoyannopoulos and Z. Weng (2020). "Expanded encyclopaedias of DNA elements in the human and mouse genomes." Nature 583(7818): 699-710.

Cox, C. J., F. Choudhry, E. Peacey, M. S. Perkinton, J. C. Richardson, D. R. Howlett, S. F. Lichtenthaler, P. T. Francis and R. J. Williams (2015). "Dietary (-)-epicatechin as a potent inhibitor of betagammasecretase amyloid precursor protein processing." Neurobiol Aging 36(1): 178-187.

Davies, J. O., A. M. Oudelaar, D. R. Higgs and J. R. Hughes (2017). "How best to identify chromosomal interactions: a comparison of approaches." Nat Methods 14(2): 125-134.

Davies, J. O., J. M. Telenius, S. J. McGowan, N. A. Roberts, S. Taylor, D. R. Higgs and J. R. Hughes (2016). "Multiplexed analysis of chromosome conformation at vastly improved sensitivity." Nat Methods 13(1): 74-80.

Derrien, T., R. Johnson, G. Bussotti, A. Tanzer, S. Djebali, H. Tilgner, G. Guernec, D. Martin, A. Merkel, D. G. Knowles, J. Lagarde, L. Veeravalli, X. Ruan, Y. Ruan, T. Lassmann, P. Carninci, J. B. Brown, L. Lipovich, J. M. Gonzalez, M. Thomas, C. A. Davis, R. Shiekhattar, T. R. Gingeras, T. J. Hubbard, C. Notredame, J. Harrow and R. Guigo (2012). "The GENCODE v7 catalog of human long noncoding RNAs: analysis of their gene structure, evolution, and expression." Genome Res 22(9): 1775-1789. ENCODE (2019). "8 Enhancer discovery and characterization." Nature. Freire-Pritchett, P., S. Schoenfelder, C. Varnai, S. W. Wingett, J. Cairns, A. J. Collier, R. Garcia-Vilchez, M. Furlan-Magaril, C. S. Osborne, P. Fraser, P. J. Rugg-Gunn and M. Spivakov (2017). "Global reorganisation of cis-regulatory units upon lineage commitment of human embryonic stem cells." Elife 6. 
Fulco, C. P., M. Munschauer, R. Anyoha, G. Munson, S. R. Grossman, E. M. Perez, M. Kane, B. Cleary, E. S. Lander and J. M. Engreitz (2016). "Systematic mapping of functional enhancer-promoter connections with CRISPR interference." Science 354(6313): 769-773.

Gerrard, D. L., Y. Wang, M. Gaddis, Y. Zhou, J. Wang, H. Witt, S. Lin, P. J. Farnham, V. X. Jin and S. E. Frietze (2019). "Three-dimensional analysis reveals altered chromatin interaction by enhancer inhibitors harbors TCF7L2-regulated cancer gene signature." J Cell Biochem 120(3): 3056-3070. Gilbert, L. A., M. A. Horlbeck, B. Adamson, J. E. Villalta, Y. Chen, E. H. Whitehead, C. Guimaraes, B. Panning, H. L. Ploegh, M. C. Bassik, L. S. Qi, M. Kampmann and J. S. Weissman (2014). "GenomeScale CRISPR-Mediated Control of Gene Repression and Activation." Cell 159(3): 647-661.

He, Y., M. Hariharan, D. U. Gorkin, D. E. Dickel, C. Luo, R. G. Castanon, J. R. Nery, A. Y. Lee, Y. Zhao, H. Huang, B. A. Williams, D. Trout, H. Amrhein, R. Fang, H. Chen, B. Li, A. Visel, L. A. Pennacchio, B. Ren and J. R. Ecker (2020). "Spatiotemporal DNA methylome dynamics of the developing mouse fetus." Nature 583(7818): 752-759.

Hezroni, H., R. Ben-Tov Perry, N. Gil, N. Degani and I. Ulitsky (2020). "Regulation of neuronal commitment in mouse embryonic stem cells by the Reno1/Bahcc1 locus." EMBO Rep 21(11): e51264.

Irastorza-Azcarate, I., R. D. Acemel, J. J. Tena, I. Maeso, J. L. Gomez-Skarmeta and D. P. Devos (2018). "4Cin: A computational pipeline for 3D genome modeling and virtual $\mathrm{Hi}-\mathrm{C}$ analyses from $4 \mathrm{C}$ data." PLoS Comput Biol 14(3): e1006030.

Jeziorska, D. M., K. W. Jordan and K. W. Vance (2009). "A systems biology approach to understanding cis-regulatory module function." Semin Cell Dev Biol 20(7): 856-862.

Jeziorska, D. M., G. Koentges and K. W. Vance (2012). "Novel cis-regulatory modules control expression of the Hairy and Enhancer of Split-1 (HES1) transcription factor in myoblasts." $\underline{\text { J Biol Chem }}$ 287(8): 5687-5697.

Jordan, T., I. Hanson, D. Zaletayev, S. Hodgson, J. Prosser, A. Seawright, N. Hastie and V. van Heyningen (1992). "The human PAX6 gene is mutated in two patients with aniridia." Nat Genet 1(5): 328-332.

Kammandel, B., K. Chowdhury, A. Stoykova, S. Aparicio, S. Brenner and P. Gruss (1999). "Distinct cisessential modules direct the time-space pattern of the Pax6 gene activity." Dev Biol 205(1): 79-97.

Kleinjan, D. A., A. Seawright, A. J. Childs and V. van Heyningen (2004). "Conserved elements in Pax6 intron 7 involved in (auto)regulation and alternative transcription." Dev Biol 265(2): 462-477.

Kleinjan, D. A., A. Seawright, S. Mella, C. B. Carr, D. A. Tyas, T. I. Simpson, J. O. Mason, D. J. Price and V. van Heyningen (2006). "Long-range downstream enhancers are essential for Pax6 expression." Dev Biol 299(2): 563-581.

Kleinjan, D. A., A. Seawright, A. Schedl, R. A. Quinlan, S. Danes and V. van Heyningen (2001).

"Aniridia-associated translocations, DNase hypersensitivity, sequence comparison and transgenic analysis redefine the functional domain of PAX6." Hum Mol Genet 10(19): 2049-2059.

Lewandowski, J. P., G. Dumbovic, A. R. Watson, T. Hwang, E. Jacobs-Palmer, N. Chang, C. Much, K. M. Turner, C. Kirby, N. D. Rubinstein, A. F. Groff, S. C. Liapis, C. Gerhardinger, A. Bester, P. P. Pandolfi, J. G. Clohessy, H. E. Hoekstra, M. Sauvageau and J. L. Rinn (2020). "The Tug1 IncRNA locus is essential for male fertility." Genome Biol 21(1): 237.

Li, Y., C. M. Rivera, H. Ishii, F. Jin, S. Selvaraj, A. Y. Lee, J. R. Dixon and B. Ren (2014). "CRISPR reveals a distal super-enhancer required for Sox 2 expression in mouse embryonic stem cells." PLoS One 9(12): e114485.

Lima Cunha, D., G. Arno, M. Corton and M. Moosajee (2019). "The Spectrum of PAX6 Mutations and Genotype-Phenotype Correlations in the Eye." Genes (Basel) 10(12).

Liu, S. J., T. J. Nowakowski, A. A. Pollen, J. H. Lui, M. A. Horlbeck, F. J. Attenello, D. He, J. S. Weissman, A. R. Kriegstein, A. A. Diaz and D. A. Lim (2016). "Single-cell analysis of long non-coding RNAs in the developing human neocortex." Genome Biol 17: 67.

Love, M. I., W. Huber and S. Anders (2014). "Moderated estimation of fold change and dispersion for RNA-seq data with DESeq2." Genome Biol 15(12): 550. 
Lu, L., X. Liu, W. K. Huang, P. Giusti-Rodriguez, J. Cui, S. Zhang, W. Xu, Z. Wen, S. Ma, J. D. Rosen, Z. Xu, C. F. Bartels, R. Kawaguchi, M. Hu, P. C. Scacheri, Z. Rong, Y. Li, P. F. Sullivan, H. Song, G. L. Ming, Y. Li and F. Jin (2020). "Robust Hi-C Maps of Enhancer-Promoter Interactions Reveal the Function of Non-coding Genome in Neural Development and Diseases." Mol Cell 79(3): 521-534 e515.

Manuel, M. N., D. Mi, J. O. Mason and D. J. Price (2015). "Regulation of cerebral cortical neurogenesis by the Pax6 transcription factor." Front Cell Neurosci 9: 70.

McBride, D. J., A. Buckle, V. van Heyningen and D. A. Kleinjan (2011). "DNasel hypersensitivity and ultraconservation reveal novel, interdependent long-range enhancers at the complex Pax6 cisregulatory region." PLoS One 6(12): e28616.

Molina-Holgado, F., A. Rubio-Araiz, D. Garcia-Ovejero, R. J. Williams, J. D. Moore, A. Arevalo-Martin, O. Gomez-Torres and E. Molina-Holgado (2007). "CB2 cannabinoid receptors promote mouse neural stem cell proliferation." Eur J Neurosci 25(3): 629-634.

Morgan, R. (2004). "Conservation of sequence and function in the Pax6 regulatory elements." Trends Genet 20(7): 283-287.

Morin, P. J., A. B. Sparks, V. Korinek, N. Barker, H. Clevers, B. Vogelstein and K. W. Kinzler (1997).

"Activation of beta-catenin-Tcf signaling in colon cancer by mutations in beta-catenin or APC."

Science 275(5307): 1787-1790.

Pavlaki, I., F. Alammari, B. Sun, N. Clark, T. Sirey, S. Lee, D. J. Woodcock, C. P. Ponting, F. G. Szele and K. W. Vance (2018). "The long non-coding RNA Paupar promotes KAP1-dependent chromatin changes and regulates olfactory bulb neurogenesis." EMBO J 37(10).

Plaza, S., S. Saule and C. Dozier (1999). "High conservation of cis-regulatory elements between quail and human for the Pax-6 gene." Dev Genes Evol 209(3): 165-173.

Ponjavic, J., P. L. Oliver, G. Lunter and C. P. Ponting (2009). "Genomic and transcriptional colocalization of protein-coding and long non-coding RNA pairs in the developing brain." PLoS Genet 5(8): e1000617.

Ritter, N., T. Ali, N. Kopitchinski, P. Schuster, A. Beisaw, D. A. Hendrix, M. H. Schulz, M. MullerMcNicoll, S. Dimmeler and P. Grote (2019). "The IncRNA Locus Handsdown Regulates Cardiac Gene Programs and Is Essential for Early Mouse Development." Dev Cell 50(5): 644-657 e648.

Singer, R. A., L. Arnes, Y. Cui, J. Wang, Y. Gao, M. A. Guney, K. E. Burnum-Johnson, R. Rabadan, C. Ansong, G. Orr and L. Sussel (2019). "The Long Noncoding RNA Paupar Modulates PAX6 Regulatory Activities to Promote Alpha Cell Development and Function." Cell Metab 30(6): 1091-1106 e1098. Szemes, M. Z. Melegh, J. Bellamy, A. Greenhough, M. Kollareddy, D. Catchpoole and K. Malik (2020). "A Wnt-BMP4 Signaling Axis Induces MSX and NOTCH Proteins and Promotes Growth Suppression and Differentiation in Neuroblastoma." Cells $\mathbf{9}(3)$.

Thongjuea, S., R. Stadhouders, F. G. Grosveld, E. Soler and B. Lenhard (2013). "r3Cseq: an $\mathrm{R} /$ Bioconductor package for the discovery of long-range genomic interactions from chromosome conformation capture and next-generation sequencing data." Nucleic Acids Res 41(13): e132.

Vance, K. W., S. N. Sansom, S. Lee, V. Chalei, L. Kong, S. E. Cooper, P. L. Oliver and C. P. Ponting (2014). "The long non-coding RNA Paupar regulates the expression of both local and distal genes." EMBO J 33(4): 296-311.

Williams, S. C., C. R. Altmann, R. L. Chow, A. Hemmati-Brivanlou and R. A. Lang (1998). "A highly conserved lens transcriptional control element from the Pax-6 gene." Mech Dev 73(2): 225-229.

Xu, P. X., X. Zhang, S. Heaney, A. Yoon, A. M. Michelson and R. L. Maas (1999). "Regulation of Pax6 expression is conserved between mice and flies." Development 126(2): 383-395.

Xu, Y., J. Xi, G. Wang, Z. Guo, Q. Sun, C. Lu, L. Ma, Y. Wu, W. Jia, S. Zhu, X. Guo, S. Bian and J. Kang (2021). "PAUPAR and PAX6 sequentially regulate human embryonic stem cell cortical differentiation." Nucleic Acids Res 49(4): 1935-1950.

Yin, Y., P. Yan, J. Lu, G. Song, Y. Zhu, Z. Li, Y. Zhao, B. Shen, X. Huang, H. Zhu, S. H. Orkin and X. Shen (2015). "Opposing Roles for the IncRNA Haunt and Its Genomic Locus in Regulating HOXA Gene Activation during Embryonic Stem Cell Differentiation." Cell Stem Cell 16(5): 504-516. 
bioRxiv preprint doi: https://doi.org/10.1101/2021.05.18.442939; this version posted May 18, 2021. The copyright holder for this preprint (which

was not certified by peer review) is the author/funder, who has granted bioRxiv a license to display the preprint in perpetuity. It is made available under aCC-BY-NC-ND 4.0 International license.

Yochum, G. S., C. M. Sherrick, M. Macpartlin and R. H. Goodman (2010). "A beta-catenin/TCFcoordinated chromatin loop at MYC integrates 5' and 3' Wnt responsive enhancers." Proc Natl Acad Sci U S A 107(1): 145-150. 
A

Figure 1

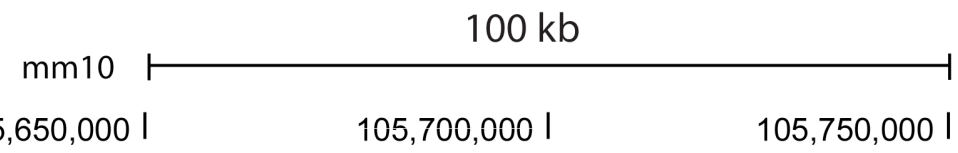

$105,800,000$ I

Chr2: $\quad 105,650,000$ I
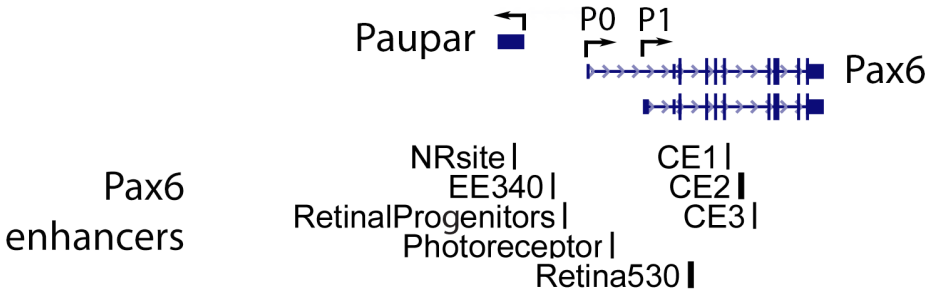

SIMOI HS6I

HS1 HS7 I

HS2 I HS8 I

HS3I
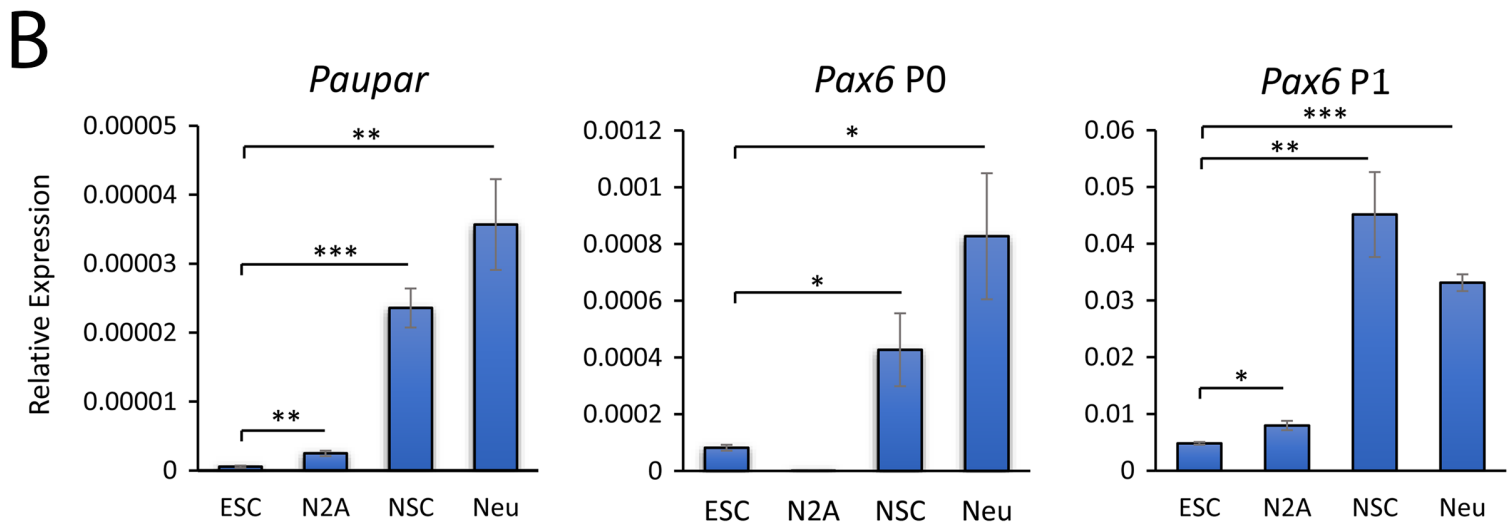


\section{Figure 3}

A

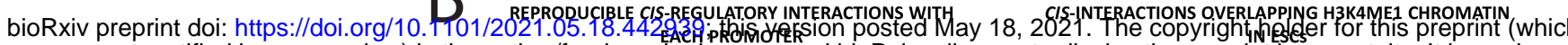

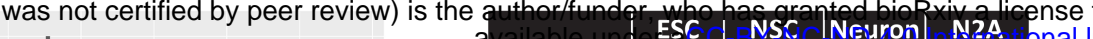

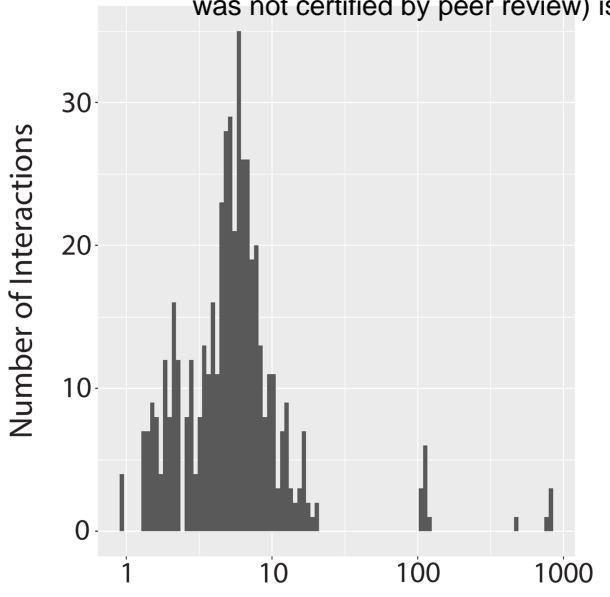

Distance in kb from fragment to viewpoint (log scale)

NSCS

Paupar

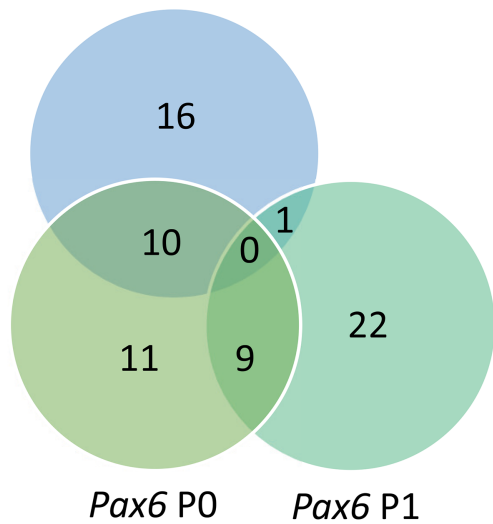

Pax6 P0 Pax6 P1

\begin{tabular}{|l|l|l|l|l|}
\hline Paupar TSS & 49 & 27 & 40 & 44 \\
\hline Pax6 P0 & 33 & 30 & 28 & 30 \\
\hline Pax6 P1 & 42 & 32 & 34 & 27 \\
\hline
\end{tabular}

CIS-INTERACTIONS CORRESPONDING TO KNOWN PAX6 LOCAL ENHANCER

\begin{tabular}{|l|c|c|c|c|}
\hline & ESC & NSC & Neuron & N2A \\
\hline Paupar TSS & 5 & 4 & 5 & 5 \\
\hline Pax6 P0 & 6 & 8 & 6 & 6 \\
\hline Pax6 P1 & 6 & 5 & 4 & 5 \\
\hline
\end{tabular}

CIS-INTERACTIONS OVERLAPPING H3K4ME1 CHROMATIN IN THE BRAIN

\begin{tabular}{|l|c|c|c|c|}
\hline & ESC & NSC & Neuron & N2A \\
\hline Paupar TSS & 22 & 10 & 14 & 16 \\
\hline Pax6 P0 & 23 & 21 & 18 & 20 \\
\hline Pax6 P1 & 34 & 26 & 29 & 20 \\
\hline
\end{tabular}

\section{Neurons}

Paupar

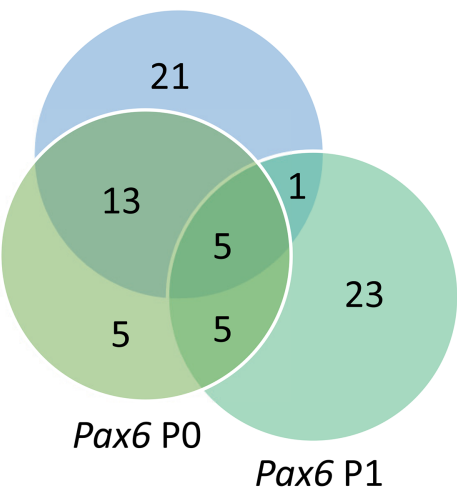

Pax6 P1

\begin{tabular}{|l|l|l|l|l|}
\hline Paupar TSS & 4 & 1 & 4 & 5 \\
\hline Pax6 P0 & 6 & 5 & 6 & 5 \\
\hline Pax6 P1 & 8 & 8 & 8 & 5 \\
\hline
\end{tabular}

CIS-INTERACTIONS BETWEEN PAUPAR LOCUS AND INDICATED PROMOTERS

\begin{tabular}{|l|c|c|c|c|}
\hline & ESC & NSC & Neuron & N2A \\
\hline Paupar TSS & 2 & 2 & 2 & 2 \\
\hline Pax6 P0 & 0 & 3 & 1 & 1 \\
\hline Pax6 P1 & 1 & 0 & 0 & 0 \\
\hline
\end{tabular}

REPRODUCIBLE TRANS INTERACTIONS WITH DNA SEQUENCES ON OTHER CHROMOSOMES

\begin{tabular}{|l|c|c|c|c|}
\hline & ESC & NSC & Neuron & N2A \\
\hline Paupar TSS & 4 & 5 & 5 & 0 \\
\hline Pax6 P0 & 5 & 4 & 4 & 0 \\
\hline Pax6 P1 & 5 & 6 & 7 & 0 \\
\hline
\end{tabular}

N2A Cells

Paupar

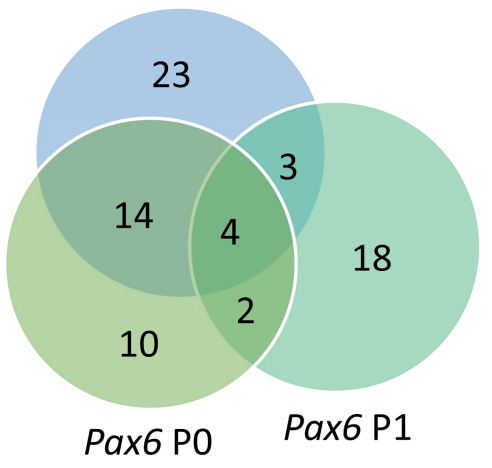

Pax6 P1 Viepwpoint

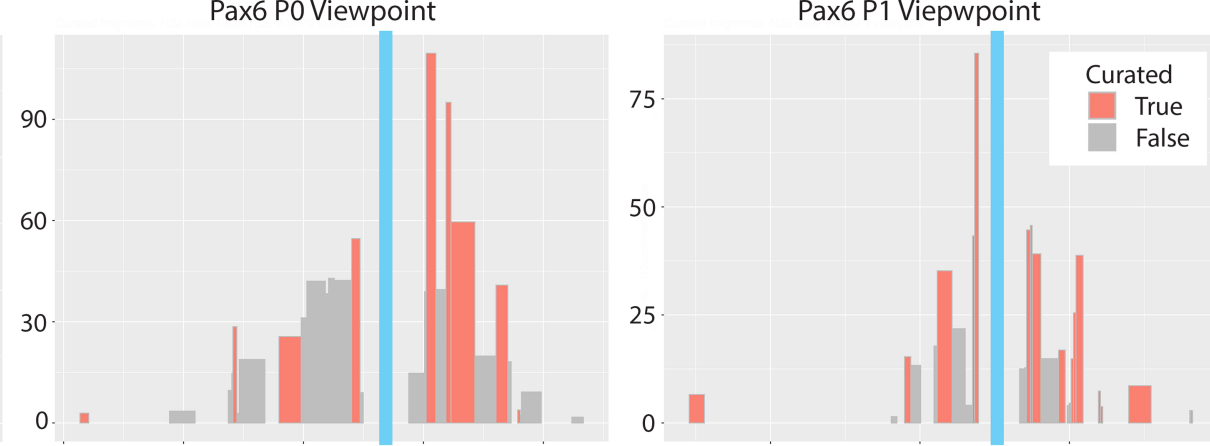

$105660000 \quad 105670000 \quad 105680000$

E
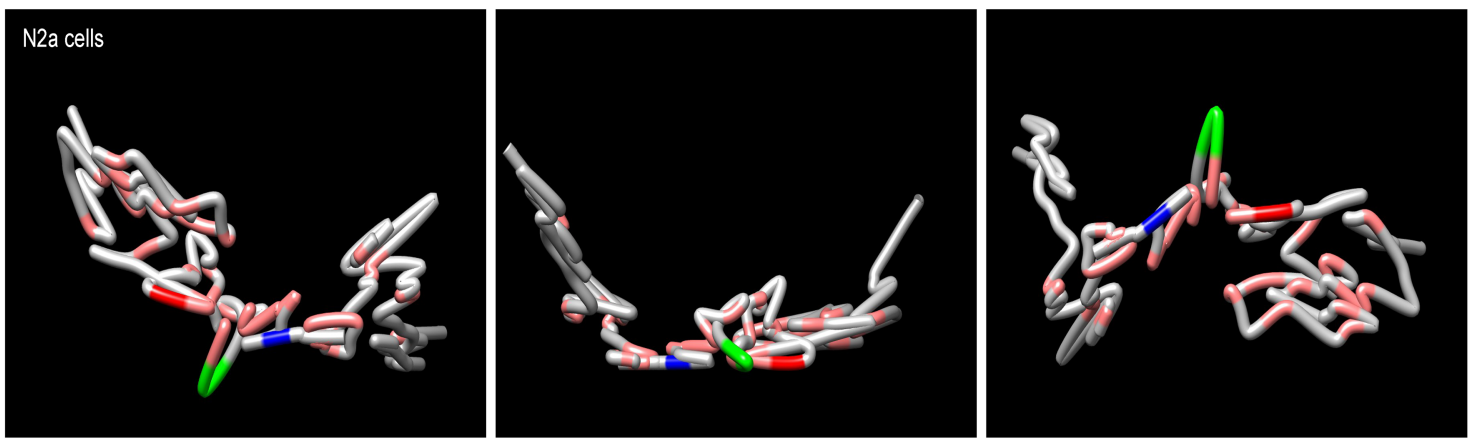

Paupar viewpoint 
Paupar ESC - Neurons $(p<0.05)$

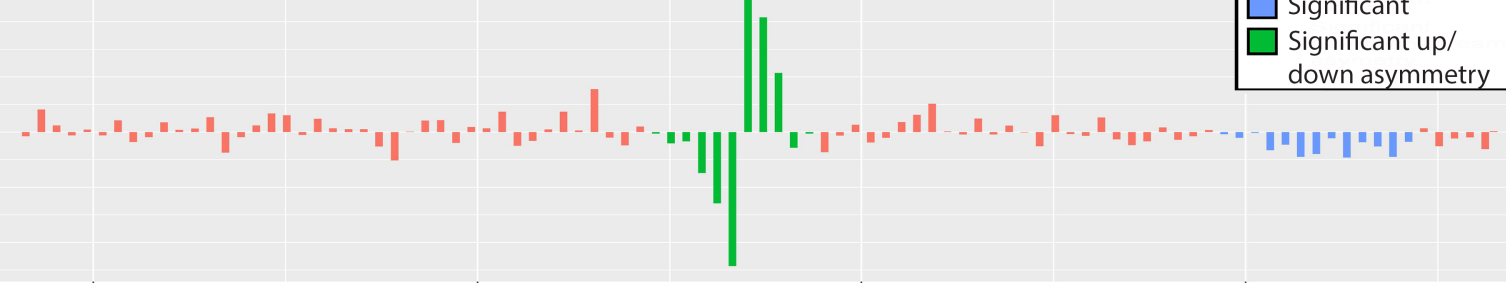

500

ن

Pax6 P0 ESC - Neurons $(p<0.05)$

$-100$

400

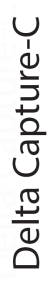

Pax6 P1 ESC - Neurons $(p<0.05)$

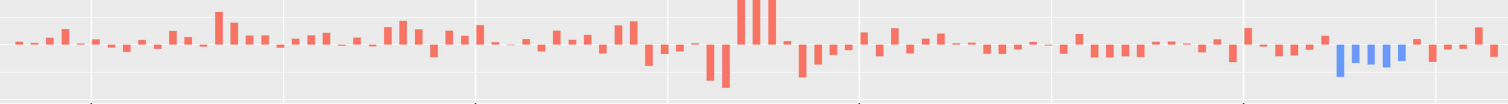

105250000

105500000
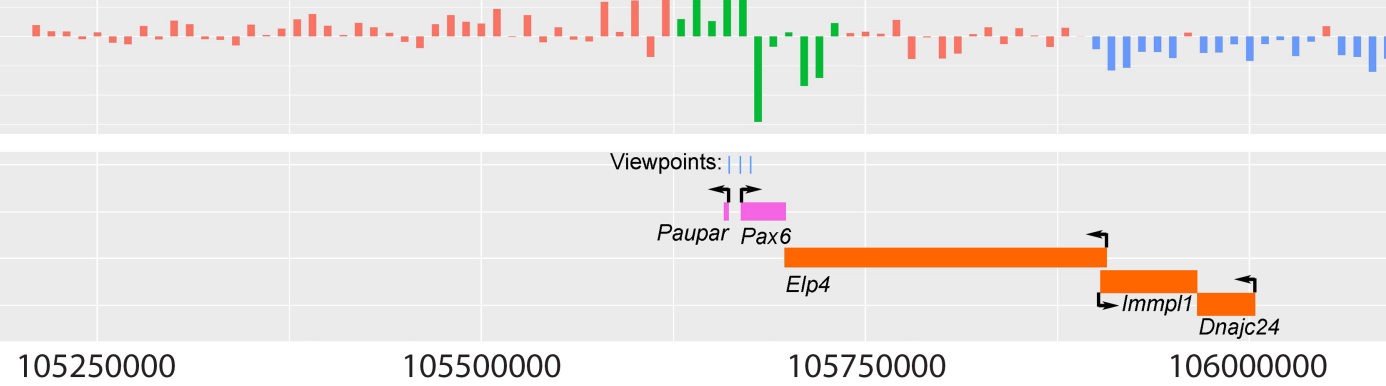

Chromatin marks used to define active regulatory regions

\section{ESC H3K4me1}

\section{ESC H3K4me3}

\section{ESC H3K27ac}

\section{Neuron H3K4me1}

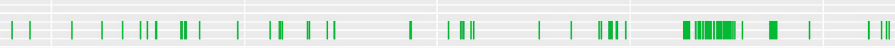

\section{Neuron H3K4me3}

\section{Neuron H3K27ac}

$\|$

Distal region of increased chromatin interactions in neurons

\section{Known Pax6 Enhancers}




\section{Supplemental Figure 1}

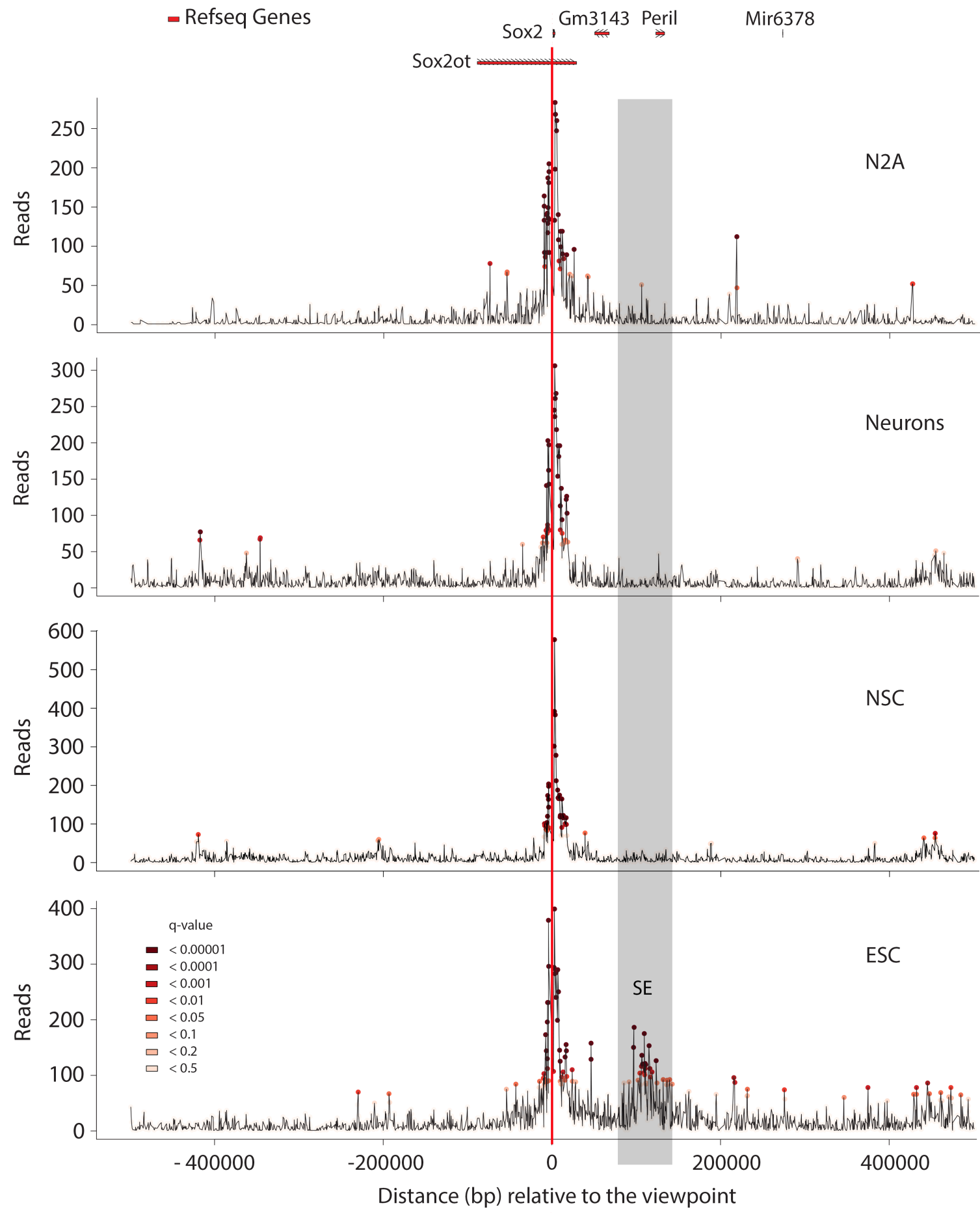


A Embryonic stem cells open / closed histone marks

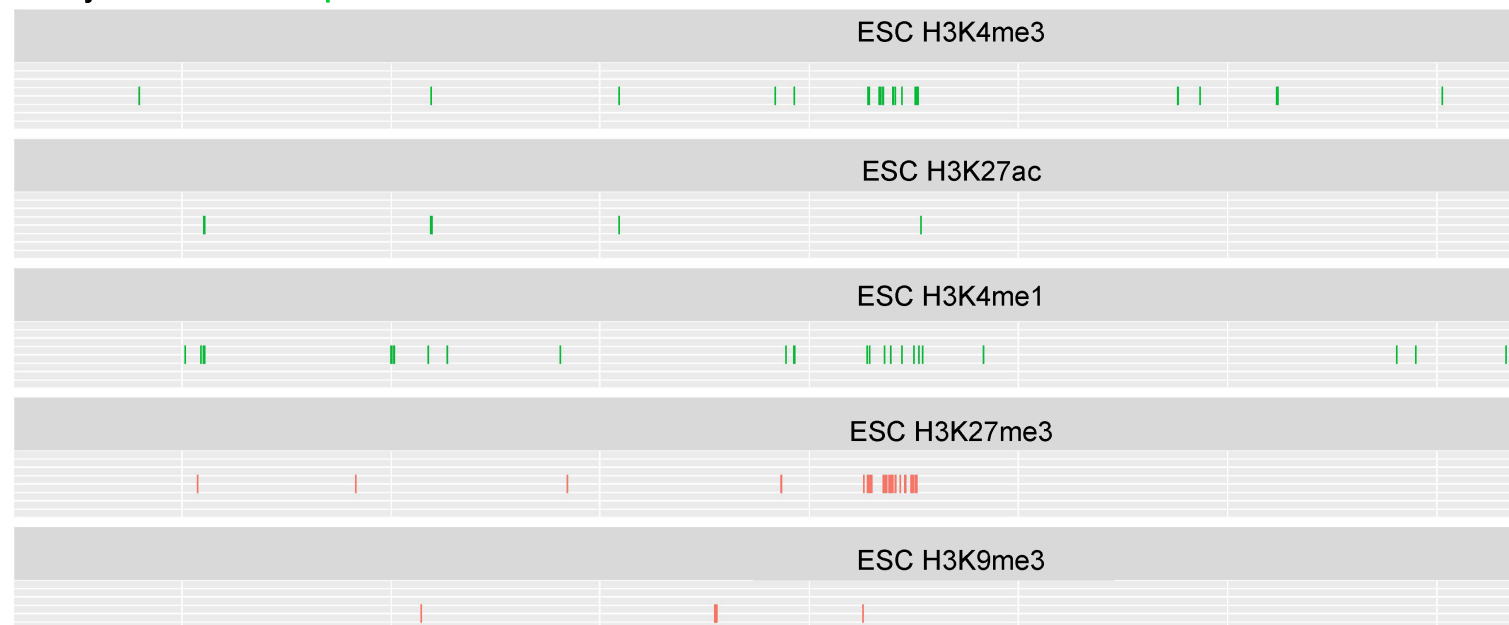

Aggregate ESC scores (binned at $\mathbf{5 0 ~ k b ) ~}$

Open Chromatin

Closed Chromatin

B Neurons open / closed histone marks

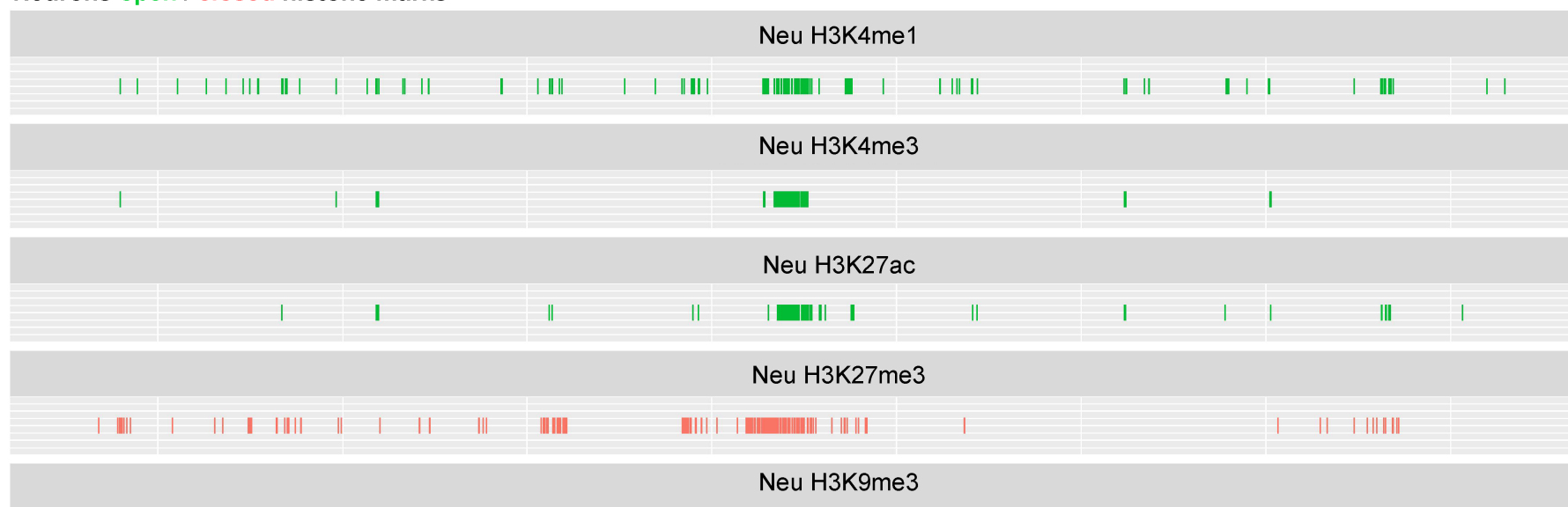

Aggregate Neu scores (binned at $50 \mathrm{~kb}$ )

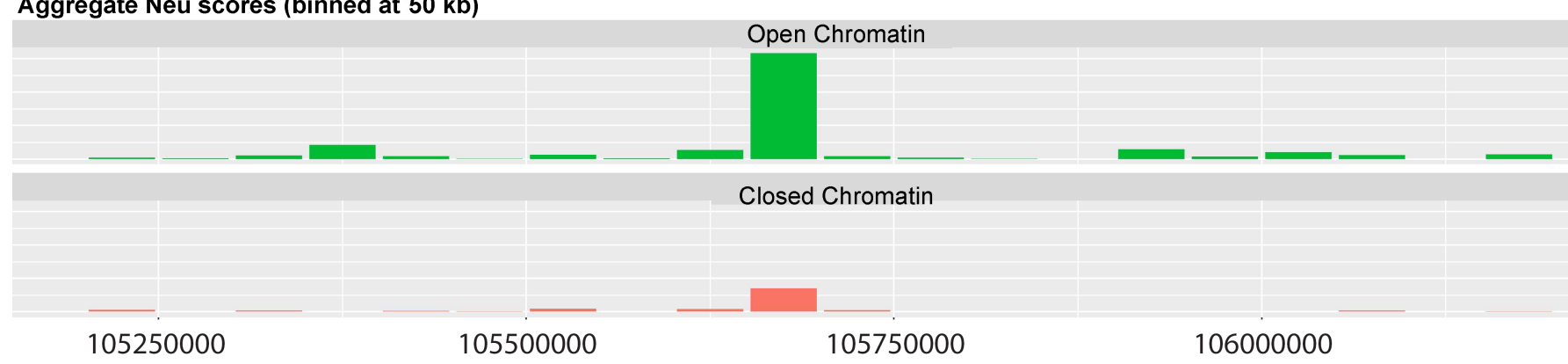

Chromosome 2 (mm10) 